\title{
Ethnic differences in antipsychotic treatment responses in early intervention in psychosis
}

\author{
ABSTRACT \\ Background \\ Ethnic variations in pathways to early intervention in psychosis (EIP) have \\ been studied. However it is important to explore the ethnic variations in \\ response to pharmacological treatment in EIP. \\ Aims \\ To look at ethnic variation in response to antipsychotics. \\ Method \\ Electronic patient records in December 2018 were perused for: \\ 1. Sociodemographic details including ethnicity \\ 2. Prescribed antipsychotic and dosage to which the patient had \\ responded \\ Results \\ White, Black and Asian ethnic groups responded mostly to Olanzapine. The \\ largest proportion of the mixed ethnic group responded to Aripiprazole. The \\ White and Asian groups showed the best responses to Lurasidone at $74 \mathrm{mg}$. \\ There was a slight response in the mixed and other ethnic group only at the \\ higher dose of $111 \mathrm{mg}$. The medication has not been used with any benefit \\ in the Black ethnic group.

\section{Conclusion} \\ The complex differences highlighted amongst ethnic responses to treatment \\ make a case for multi-centric studies to further explore ethnic differences in \\ early intervention treatments. This will help streamline offered treatments \\ to maximise response in EIP.
}

\section{Keywords}

Ethnicity; antipsychotic medication; early intervention in psychosis

\section{Introduction}

People who have experienced a psychotic illness have experienced long term benefits from engaging with early intervention in psychosis services. These services have been more effective when the intervention is made earlier, making the duration of untreated psychosis (DUP) shorter. The DUP is understood to end with the initiation of antipsychotic medication.[1]

Ethnic differences in response to antipsychotic medications have been reported.[2] Some studies have backed this up, suggesting that ethnicity may play a role in symptom presentation and treatment response to antipsychotics. $[3,4]$ In contrast, one study did not find any ethnic variation in response to the antipsychotic clozapine [5] whilst another study has found no differences in the pharmacokinetics of dosage requirements of antipsychotic drugs amongst Black, Hispanic, and White ethnic groups.
Professor Nandini Chakraborty, Shiraz Ahmed, Hanna Booth \& Hawa Aswat

\section{PIER team, Leicestershire Partnership NHS Trust, Merlyn Vaz Health Centre, Leicester, UK LE5 $3 G H$ \\ Correspondence to nandinichakraborty@doctors.org.u $\underline{\mathrm{k}}$}

Cite as: Chakraborty, N., Ahmed S., Booth, H., Aswat, H. (2021) Ethnic differences in antipsychotic treatment responses in early intervention in psychosis. The Physician vol 7; Issue 1: 1-8 ePub 9.5.21 DOI https://doi.org/10.38192/1.7.1.6
Article Information

Submitted 6.5.21

ePub 9.5.21

ISSN 2732-513X (Print) ISSN 2732 - 5148 (Online) 
However the same study demonstrated that the Asian ethnic group have lower therapeutic and side effect thresholds

compared to the White ethnic group.[6] A prolonged DUP may indicate the need for higher doses of antipsychotics, but ethnicity has been seen not to be a factor in determining DUP. [7] With respect to variations in prescribing practices which are based on ethnic variations, some studies did find that such differences did exist. [8,9] Whilst other studies showed that antipsychotic prescribing practice did not differ between patients from the Black and White ethnic groups.[10,11]

The Psychosis Intervention Early Recovery (PIER) team is the Early intervention in Psychosis team which covers the county of Leicestershire, with a populace numbering 696,742.[12] Leicester, being the principal city in the area, comprises a multiethnic population. The White ethnic group are the largest ethnic group in the city with 50.6 percent of the total population.[13] These represent various places of origin: there are 45.1 percent British whites, 0.8 percent Irish whites and 4.6 percent white people from other places. Other groups include the Black ethnic group (6.3 percent), the Asian ethnic groups (37.1 percent), the Arab ethnic group (1.6 percent) and other groups (1.6 percent). Besides Loughborough, the other towns in the county contain predominantly people from the White ethnic group. It is important for the PIER team to address any ethnic variations of positively responding to antipsychotic medication, given the diverse ethnic background of patients on the caseload.

This study aimed to look at ethnic variations in response to antipsychotics- both type and dosage.

\section{Method}

Due to the diverse and varied nature of ethnicity, it was essential to categorise ethnicities into broader categories that would encompass and allow for meaningful comparisons, which are the following:

- White (includes White British, White Irish, and White Other)

- Asian (includes Asian British, Asian Pakistani, Asian Indian, and Chinese)

- Black (Includes Black British, Black Caribbean, Black African, and Other Black Background)

- Mixed (includes Black Caribbean and White, Asian and white).

The study was retrospective and cross-sectional in design. The population of the study was represented by case load of the PIER (Psychosis intervention and early recovery, the EIP team for Leicestershire
Partnership NHS Trust) team on 11th December 2018.

The inclusion criteria were:

- The patients on the caseload of the PIER team on 11 December 2018.

- Patients whose psychotic symptoms were considered remitted to a degree where they no longer fulfilled diagnostic criteria for a psychotic episode.

The exclusion criteria were:

- Patients who continued to have active psychotic symptoms sufficient to fulfil criteria for a psychotic episode.

- Patients who reported psychotic symptoms which were assessed to be not due to a psychotic illness, for example borderline personality disorder.

Each patient's electronic notes were perused to determine if their psychotic symptoms were resolved to the point that the patient no longer fulfilled diagnostic criteria for a psychotic episode. The antipsychotic medication and the dose the patient was taking at the time of initial remission of psychotic symptoms, was recorded. The data was then filtered to remove patients who were on the caseload, but their symptoms were later judged not be based on a psychotic illness. Then those patients whose psychosis remained unresolved, or whose symptoms went into remission without the use on an antipsychotic, were screened out.

The numbers of patients on each antipsychotic medication was then compiled, according to each ethnic group. To enable a standardised comparison between the responses of each ethnic group to the antipsychotics, the proportion of patients from each ethnic group responding to an antipsychotic was calculated as a percentage of the total number of patients who responded to all the antipsychotics from that ethnic group.

To determine any patterns of variations of doses of an antipsychotic that the ethnic groups responded to, line graphs were generated to identify any trends within an ethnic group and its response to the dose of a medication, and to compare trends between ethnic groups. The antipsychotics which patients responded to infrequently were omitted. A compound bar chart was made to show the percentage breakdown of which antipsychotic resulted in remission for each ethnic group. Then line graphs were generated for antipsychotics showing the proportion of patients from each ethnic group 
that responded to a particular dose of that antipsychotic.

The data that support the findings of this study are available on request from the corresponding author. The data are not publicly available due to privacy or ethical restrictions.

\section{Results}

A total of 455 patients were screened. Table 1 shows the breakdown of the caseload by the different ethnic groups. The distribution of ethnicity in the county population is largely reflected the PIER team case load, with the White ethnic group being the most prominent, and actually equal to that in the county, at $46 \%$. The Asian ethnic group is the next highest, but is under represented in the PIER caseload at $23 \%$ as opposed to $37 \%$ in the community. The Black ethnic group is slightly over represented in the PIER case load at $10 \%$, as opposed to $6 \%$ in the community. The other and mixed ethnic group has a high representation in the PIER case load at $21 \%$. Given that the "other ethnic group" accounts for about $3 \%$ of the community, is can be inferred that the $21 \%$ is made up of a majority of mixed ethnic groups. Table 2 shows the breakdown of patients who were not applicable to the study and those who responded to medication. The Black ethnic group showed the best over response, with $93 \%$ of this group responding favourably to an antipsychotic, compared to $81 \%$ for the other groups.

Table 1 Breakdown of the caseload by the different ethnic groups

\begin{tabular}{|l|l|l|}
\hline Ethnicity & N & $\%$ \\
\hline White & 210 & 46 \\
\hline Asian & 104 & 23 \\
\hline Black & 46 & 10 \\
\hline Mixed/Other & 95 & 21.0 \\
\hline Total & 455 & \\
\hline
\end{tabular}

Table 2 Breakdown by Ethnicity of Patients Psychosis Resolving with an Antipsychotic

\begin{tabular}{|l|l|l|l|l|}
\hline Ethnicity & $\begin{array}{l}\text { Not } \\
\text { Applicable }\end{array}$ & Resolved & Unresolved & $\begin{array}{l}\text { Percentage } \\
\text { Resolved }\end{array}$ \\
\hline White & 14 & 160 & 36 & 81.6 \\
\hline Asian & 2 & 83 & 19 & 81.4 \\
\hline Black & 3 & 40 & 3 & 93.0 \\
\hline Mixed/Other & 13 & 65 & 17 & 79.3 \\
\hline Total & 32 & & & 82.3 \\
\hline
\end{tabular}

Figure 1 is a column graph shows the percentage breakdown of which antipsychotic each ethnic group responded to. A rough comparison shows an even distribution of most of the antipsychotic medication across each ethnic group. The biggest noticeable difference was shown by the Black ethnic group having a proportionately larger response to olanzapine, when compared to the other groups. For the White, Black and Asian ethnic groups, Olanzapine was the medication that the largest proportion that each group responded to. This was most prominent in the Black group with almost $50 \%$ of these patients responding well to Olanzapine. This would coincide with it being the most used antipsychotic. The largest proportion of the mixed ethnic group responded to Aripiprazole, which is widely used as a first line antipsychotic in PIER. In exploration of which dose of an antipsychotic an ethnic group responded to, the line graphs in figures 2,3 and 4 separated the percentage breakdown of which antipsychotic each ethnic group responded to by the dose for each medication. 
Figure 2 combines Olanzapine and Aripiprazole, which showed the largest proportion of all ethnic groups responded to. With regards to Olanzapine, the Black ethnic group showed the majority of their responses to doses of $10 \mathrm{mg}$ or less. For the other ethnic groups, there was no difference in responses to Olanzapine across the different doses.

For Aripiprazole, a pattern emerges across the different ethnic groups of high proportion of patients responding to lower doses of this medication, with a trend of the proportion getting lower as the dose becomes higher. The Asian ethnic group is different in this respect with its higher proportion of response being equally at the lowest and at the highest dose of the tablet form of Aripiprazole, and on the $400 \mathrm{mg}$ depot form . The highest proportion of for the Black and White groups for their response to Aripiprazole comes at $400 \mathrm{mg}$, the depot version of this medication.

Responses to Risperidone and its metabolite in the injectable form, Paliperidone, are shown in figure 3. There are marked differences in each ethnic group to this medication. The Black ethnic group has only shown a response to the tablet form of Risperidone in higher dose of $6 \mathrm{mg}$. The Asian ethnic group had a bimodal distribution in its responses to Risperidone, with its best responses being at $2 \mathrm{mg}$ and $6 \mathrm{mg}$, whereas the White ethnic group had its best response at $3 \mathrm{mg}$. The mixed any other ethnic group did not show any significant respond to Risperidone.
The general trend for the ethnic groups' responses to the Paliperidone was that there were better responses to the medication at higher dose. This would seem to be due the dosing regimen that is required for this medication, with a high loading dose, then a therapeutic dose tending to closer to this dose.

With reference to figure 4 , there is a there is a marked variation in the proportion of patients from each ethnic group that have responded to Quetiapine. The mixed and other ethnic groups did not respond to this at all. The Black ethnic group only showed a small proportionate response at a middle dose. An equivalent proportion of the White ethnic group showed responses to Quetiapine at a dose of $300 \mathrm{mg}$ and lower. At higher doses the proportionate response is even lower. Of note is the Asian ethnic group having a higher proportionate response to Quetiapine at the lowest doses.

Lurasidone is a newer antipsychotic and it has not been used as frequently as the other antipsychotics which have been analysed in this section. But its frequency of use in the PIER team has surpassed the use of other older and well known medications such as Amisulpride and the first generation antipsychotics. Referring again to figure 4, The White and Asian groups showed the best responses to Lurasidone at $74 \mathrm{mg}$. There was a slight response in the mixed and other ethnic group only at the higher dose of $111 \mathrm{mg}$. The medication has not been used with any benefit in the Black ethnic group.

Figure 1 Compound column graph shows the percentage breakdown of the antipsychotic each ethnic group responded to.

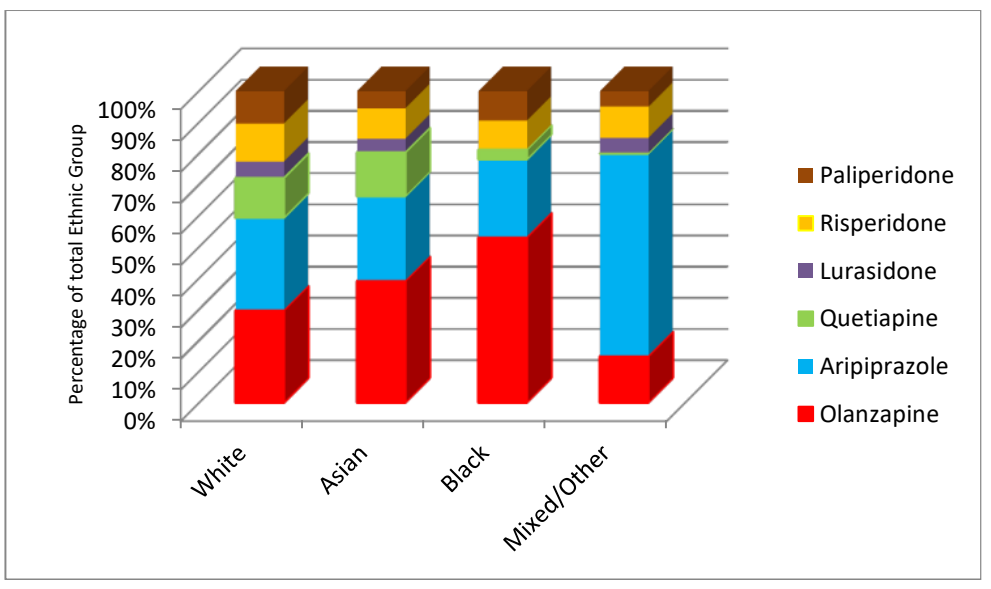

Figure 2 Line Graph Showing the Proportion of Patients from each Ethnic Group that Responded to different antipsychotics -Olanzepine and Aripiprazole. 
THE PHYSICIAN

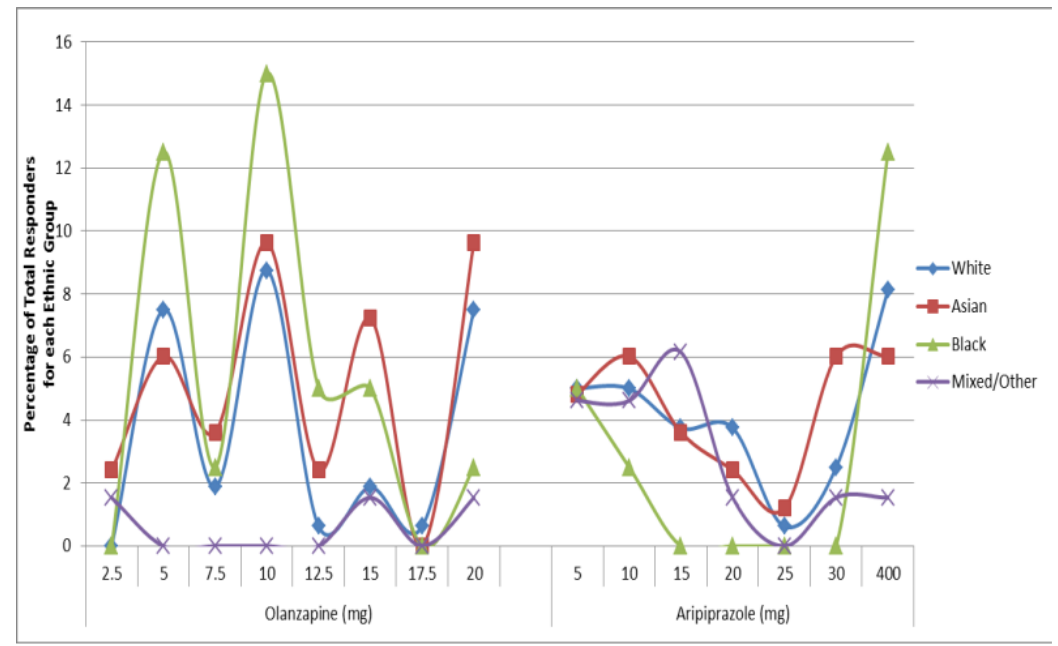

Figure 3: Line Graph Showing the Proportion of Patients from each Ethnic Group that Responded to different doses of Risperidone and Paliperidone.

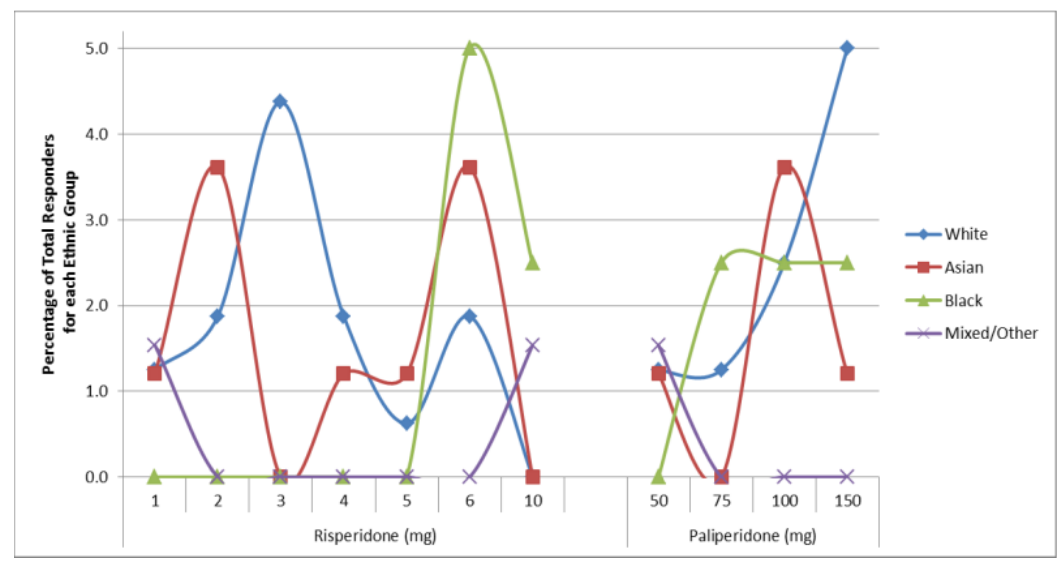

Figure 4: Line Graph Showing the Proportion of Patients from each Ethnic Group that Responded to different doses of Quetiapine and Lurasidone.

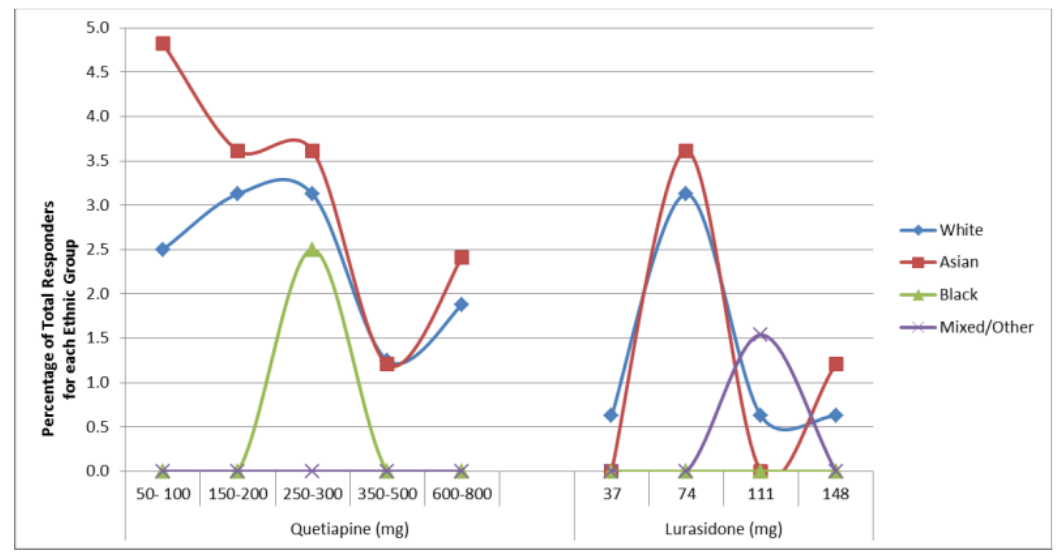

Discussion

The differential response to pharmacological interventions in different ethnicities

Physicianjnl.net | Vol 7 | Issue 1 | May 2021 | Chakraborty, N. et. al. Ethnic differences in antipsychotics

5 
Research has shown the likelihood that racial and ethnic differences in response to pharmacology [14] are important factors to consider during treatment. Whilst this may be influenced by lifestyle, diet and understanding of the treatment from cultural perspectives of both the patient and the physician, there are also genetic factors which influence metabolism and drug receptors. Pharmacogenetics is the study of genetically determined variations in drug response. Variations in drug response are caused by gene polymorphisms, leading to changes in proteins such as a drug metabolizing enzyme or a drug receptor. Burroughs et al concluded that treatment planning should take into account known ethnicityrelated variations in response to medication.

\section{Ethnic differences in treatment outcomes in psychiatry}

As with other fields, increasingly it is becoming apparent that ethnicity is an influencing factor in responses to psychotropic medication, particularly in the context of anxiety and depression [15][16] which is hypothesised to relate to both genetic factors [17] and other variables such as cultural attitudes and dietary variations. [15] It has also been suggested that different approaches should be adopted in the engagement of patients from differing cultures with respect to psychopharmacological treatments.[18]

\section{Ethnic differences in treatment outcomes in psychosis}

Research has also begun to investigate treatment responses in psychosis and their relationship to ethnicity and genetics. An early American study suggested that differences in treatment responses in Asians may be due to differences in pharmacodynamic and pharmacokinetic responses, causing them to require lower doses of antipsychotics.[2]

This was complimented by a later study showing poorer treatment responses and treatment adherence to risperidone in black youths with schizophrenia spectrum disorders compared with their white counterparts. [3] Similarly, Han Chinese patients with a first episode of schizophrenia have been shown to require lower doses of ziprasidone to treat symptoms than Mongolian patients. Mongolian patients in the study had statistically significantly greater weight gain and increases in BMI whilst taking ziprasidone.[4]

However a similarly sized study comparing antipsychotic dosing of all antipsychotics using chlorpromazine-equivalent calculations in white Europeans compared to non-white Europeans found no significant differences using both self -reported ethnicity and geographical ancestry determined by DNA sample.[7]

The contradictions between these studies could perhaps be explained by the different approaches: homing in on individual medications sharpens the focus on ethnic differences in response to that treatment which is lost when comparing with many other drugs all at once. Alternatively given that the interplay between genes and environment is notoriously complex, there may also be differences in European responses compared with those from other continents.

Recent studies have focussed more specifically on genetics. Significant associations have been found with a variety of genes associated with varying responses to clozapine, risperidone, quetiapine and chlorpromazine, and a recent Chinese study identified further candidate genes.[19]

\section{Variations in Prescribing and Treatment Pathways for Different Ethnic Groups}

In addition, many existing studies have focussed on treatment pathways and accessibility to services which have found inequalities amongst ethnic groups. This is of course influenced by the variety of cultural contexts and health care systems within which studies are completed. A recently study [20] reported that relative to white service users, ethnic minority service users with psychosis in the UK were generally less likely to be offered a range of evidence-based treatments for psychosis, which included pharmacological and psychological interventions as well as involvement in care planning. Compared with white service users, black service users were more likely prescribed depot/injectable antipsychotics. Black service users with treatment resistance were less likely to be prescribed clozapine.

However, a study [10] looked at the quality of inpatient prescribing in three South London mental health trusts and found no differences between black and white patients. Nor were there any statistical differences in a later multicentre study published by the same author.[11] Further research is indicated to clarify the differences between these studies which could be explained by changes over time, given the 710 year gap between them.

In the US, a 2004 study [9] showed that African Americans were less likely to be prescribed second generation antipsychotics. They are also less likely to be prescribed clozapine [6] in spite of there being no identified ethnic differences in its effectiveness [5]. 
Latino Medicaid beneficiaries are less likely than their white counterparts to be prescribed long acting risperidone. Thus there is significant research indicating prescribing variations on both sides of the Atlantic.

Guidelines for Practice:

NICE guidelines (National Institute for Health and Care Excellence, 2014) recommend the use of atypical antipsychotics as a first line of treatment for psychosis. The particular antipsychotic used is not specified and may depend on prescribing practices; other than in the case of clozapine where the protocols are more particular.

\section{Limitations of this Study}

It is important to disentangle prescribing practices from perceived effectiveness of the antipsychotic. We have detailed our results from patients who have responded to a particular antipsychotic medication. However whether they might have responded to other antipsychotic medication remains a matter of debate.

Nor have we been able, within the confines of this study, to identify the thought processes behind our prescribing practices since clinical details have not been included. For example, olanzapine may be considered for a young, slim individual showing signs of aggression but avoided in a person with a family history of diabetes, perhaps with lower risk symptoms who is who is afraid of being sedated or experiencing weight gain. Aripiprazole may be avoided in people who are already restless due to the risk of akathisia. Our prescribing is also within the jurisdiction of local trust guidelines who do not sanction Lurasidone as a first line treatment (Leicestershire Medicines Strategy Group 2020).[21]

Additionally our study looks at patients treated on our Early Intervention Team, but does not differentiate between medications that were started within our own service or by a referring secondary service. A study from within our team [22] identified that $45 \%$ of our patients have treatment initiated by inpatient services and $16.7 \%$ by the Crisis Resolution Team. Thus our own practices are heavily influenced by those of our prescribing predecessors.

Whether the findings really represent an ethnic variation in response to antipsychotic medication can only be verified by multi-centric research which replicates our study design in other early intervention in psychosis teams, in order to compare and assimilate findings. Further research into medical attitudes towards differing medications as well as different ethnic groups is also indicated.

\section{References}

1 Birchwood $\mathrm{M}$, Connor $\mathrm{C}$, Lester $\mathrm{H}$, et al. Reducing duration of untreated psychosis: care pathways to early intervention in psychosis services. Br J Psychiatry 2013;203:58-64. doi:10.1192/bjp.bp.112.125500

2 Frackiewicz EJ, Sramek JJ, Herrera JM, et al. Ethnicity and antipsychotic response. Ann Pharmacother 1997;31:1360-9. doi:10.1177/106002809703101114

3 Patel NC, Crismon ML, Shafer A, et al. Ethnic variation in symptoms and response to risperidone in youths with schizophreniaspectrum disorders. Soc Psychiatry Psychiatr Epidemiol 2006;41:341-6. doi:10.1007/s00127006-0036-4

4 Lv D, Zhao M, Chen L, et al. An Inter-Ethnic Comparison Study of Ziprasidone Plasma Levels, Dosage and Clinical Response in Patients with Schizophrenia. Psychiatry Investig 2017;14:3607. doi:10.4306/pi.2017.14.3.360

5 Horvitz-Lennon M, Donohue JM, Lave JR, et al. The effect of race-ethnicity on the comparative effectiveness of clozapine among Medicaid beneficiaries. Psychiatr Serv 2013;64:230-7. doi:10.1176/appi.ps.201200041

6 Singh SP, Brown L, Winsper C, et al. Ethnicity and pathways to care during first episode psychosis: the role of cultural illness attributions. BMC Psychiatry 2015;15:287. doi:10.1186/s12888015-0665-9

7 Hassan A, Teo C, Kennedy JL, et al. Association of Ethnicity with Antipsychotic Dosage Using STRUCTURE Analysis. Pharmacopsychiatry 2013;02:151-5. doi:10.1055/s-0032-1333236

8 Kuno E, Rothbard AB. Racial Disparities in Antipsychotic Prescription Patterns for Patients With Schizophrenia. AJP 2002;159:567-72. doi:10.1176/appi.ajp.159.4.567

9 Herbeck DM, West JC, Ruditis I, et al. Variations in Use of Second-Generation Antipsychotic Medication by Race Among Adult Psychiatric Patients. PS 2004;55:677-84. doi:10.1176/appi.ps.55.6.677

10 Connolly A, Taylor D. Ethnicity and quality of antipsychotic prescribing among in-patients in 
south London. Br J Psychiatry 2008;193:161-2. doi:10.1192/bjp.bp.108.050427

11 Connolly A, Taylor D, Sparshatt A, et al. Antipsychotic prescribing in Black and White hospitalised patients. J Psychopharmacol 2011;25:704-9. doi:10.1177/0269881109387841

12 Leicestershire Population. http://population.city/unitedkingdom/leicestershire/ (accessed 9 May 2021).

13 Leicester Population 2021 | Population UK. https://www.ukpopulation.org/leicesterpopulation/ (accessed 9 May 2021).

14 Burroughs VJ, Maxey RW, Levy RA. Racial and Ethnic Differences in Response to Medicines: Towards Individualized Pharmaceutical Treatment. JOURNAL OF THE NATIONAL MEDICAL ASSOCIATION 2002;94:26.

15 Ethnicity and psychopharmacology - IB Chaudhry, K. Neelam, V. Duddu, N. Husain, 2008. https://journals.sagepub.com/doi/abs/10.1177/ 0269881107082105 (accessed 9 May 2021).

16 Biological differences in depression and anxiety across races and ethnic groups. - Abstract Europe PMC. https://europepmc.org/article/MED/11434414? singleResult=true (accessed 9 May 2021).

17 Sramek JJ, Pi EH. Ethnicity and antidepressant response. Mt Sinai J Med 1996;63:320-5.

18 Malik M, Lake J, Lawson WB, et al. Culturally adapted pharmacotherapy and the integrative formulation. Child Adolesc Psychiatr Clin N Am 2010;19:791-814. doi:10.1016/j.chc.2010.08.003

$19 \mathrm{Xu} \mathrm{Q}, \mathrm{Wu}$ X, Li M, et al. Association studies of genomic variants with treatment response to risperidone, clozapine, quetiapine and chlorpromazine in the Chinese Han population. Pharmacogenomics J 2016;16:357-65. doi:10.1038/tpj.2015.61

20 Das-Munshi J, Bhugra D, Crawford MJ. Ethnic minority inequalities in access to treatments for schizophrenia and schizoaffective disorders: findings from a nationally representative crosssectional study. BMC Med 2018;16:55. doi:10.1186/s12916-018-1035-5

21 Drug Detail. Leicestershire Medicines Strategy Group. https://www.Imsg.nhs.uk/trafficlights/drug-detail/ (accessed 9 May 2021).

22 Chakraborty N, Ahmed S, Mohammed FJ. Reducing the duration of untreated psychosis: roles of mental health teams. Progress in Neurology and Psychiatry 2016;20:19-21. doi:https://doi.org/10.1002/pnp.435

Data available on request from authors Declaration of interest: none
This study was based on service evaluation from existing clinical records, which were anonymised, retrospective and within practice as usual. Approval was sought and granted by the local Trust Clinical Standards Committee and Research and Development. 